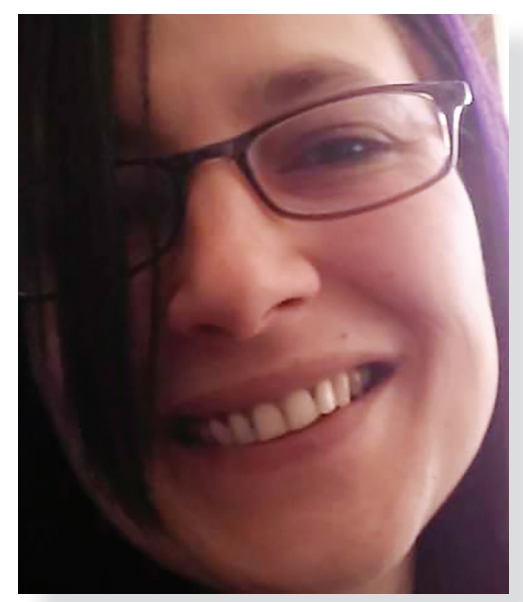

Laura Colombo*

\section{Los lectores intermedios: un recurso fundamental para la escritura de la tesis**}

\section{Proof-readers: a key resource in the $\mathrm{PhD}$ thesis writing process}

Recibido: 05-05-18

Aprobado: 31-05-18

\title{
Resumen
}

En la mayoría de las universidades de Latinoamérica, a fin de acreditar sus programas doctorales, los estudiantes deben producir un trabajo escrito en el cual dan cuenta de su proceso investigativo: la tesis doctoral. La elaboración de este tipo de texto les implica aprender a participar de prácticas sociales y discursivas determinadas. Es decir, para poder producir una tesis efectiva, los estudiantes deben aprender los modos de ser y hacer en sus respectivos campos disciplinares. Este aprendizaje se da, principalmente, mediante el intercambio con otros. En este trabajo analizamos las interacciones con otros que influencian el proceso de redacción de las tesis doctorales. Específicamente, exploramos el rol de los lectores prueba: aquellas personas que interactúan con versiones tempranas de la tesis para ofrecer feedback a los doctorandos que les permita mejorar el texto, con el propósito de valorar el aporte que ofrecen y su influencia en el proceso de redacción. Para lograr el objetivo de nuestro estudio exploratorio, utilizamos entrevistas en profundidad a 20 doctorandos y recientes doctores en el área de Educación y Lingüística. Las entrevistas fueron transcriptas y para su análisis se siguió una estrategia categorizadora y contextualizadora y se combinaron procesos inductivos y deductivos. Los resultados muestran que este es un recurso que los doctorandos utilizan con frecuencia y consideran valioso para el proceso de redacción ya que lo enriquecen.

\begin{abstract}
Accreditation of doctoral programs in most Latin American universities requires that students produce a written work based on their research: the doctoral thesis. Writing this type of text implies their involvement in specific social and discursive practices. In other words, to produce a successful doctoral thesis, students must learn the ways of doing and being in their disciplines. This learning happens, mainly, thanks to interactions with other people. This work analyzes the interactions that influence the process of writing a doctoral thesis. Specifically, the role of proof-readers (those readers who interact with early versions of the thesis and offer them feedback to improve the text) is analyzed with the aim of appraising their influence and contribution to the thesis writing process. This exploratory study was based on 20 in-depth interviews with doctoral students and recent graduates from the Education and Linguistics fields. Interviews were transcribed and analyzed by following a categorizing and contextualizing strategy and combining inductive and deductive processes. Results show that this resource is often used and highly valued by doctoral students as it enriches their writing process.
\end{abstract}

Palabras clave

borradores; lectores prueba; escritura académica; tesis

\section{Keywords}

drafts; proof-readers; academic writing; thesis

\footnotetext{
* Laura Colombo: Doctora en Lenguaje, Literacidad y Cultura y Magíster en Comunicación Intercultural. Investigadora Asistente CONICET-Instituto de Lingüística de la Universidad de Buenos Aires. Integrante del GICEOLEM (https://sites.google.com/site/ giceolem2010/). Para contactar a la autora: laura.colombo@conicet.gov.ar

${ }_{* *}^{*}$ Este trabajo forma parte del Proyecto PICT 2014/2793 financiado por la Agencia Nacional de Promoción Científica y Técnica de Argentina.
} 


\section{Introducción}

La mayoría de los programas doctorales de Latinoamérica se acreditan mediante la presentación de un trabajo escrito: la tesis. La elaboración de este tipo de texto es un proceso complejo que, a la vez, pone de relieve el rol fundamental que juega la escritura en los modos actuales y legitimados de producción de la ciencia (Canagarajah, 2002; Lee, 2013; Nygaard, 2015). Así, se espera que los estudiantes, al realizar sus tesis, dejen de ser meros receptores de textos académicos para convertirse en productores de conocimiento (Arnoux, 2009; Carlino, 2012) que participan en las conversaciones académicas de sus campos disciplinares (Aitchison, Kamler, y Lee, 2010). Sin embargo, muchos estudiantes no finalizan sus tesis de posgrado debido a las dificultades que hallan en su escritura (Ahern y Manathunga, 2004; Brunner y Ferrada Hurtado, 2011; Carlino, 2005; Elgar, 2003; Lovitts y Nelson, 2000; Lundell y Beach, 2003; Torrance y Thomas, 1992; entre otros) lo cual se refleja en la baja tasa de egreso que existe en este nivel.

En efecto, elaborar una tesis no es tarea fácil. Para ello los doctorandos deben aprender los modos de ser y hacer en sus respectivos campos de conocimiento incluyendo los usos específicos que se les dan a las prácticas de lectura y escritura (Prior, 1998). Ahora bien, ¿cómo sucede esto? Desde un enfoque sociocultural del aprendizaje (Lave y Wenger, 1991; Vygotsky, 1978; entre otros), este aprendizaje se logra mediante procesos de participación social. Por ende, aprender a escribir una tesis no constituye una "internalización" por parte del sujeto de un conocimiento objetivo sobre la escritura académica, sino que implica un proceso de participación gradual en determinados contextos sociales (Lave y Wenger, 1991; Wenger, 1998), donde el desarrollo de la identidad escritora se produce mediante la gradual participación y transformación de comunidades de práctica (Colombo, 2012). Esto incluye adentrarse en una trama compuesta de interacciones con otros sujetos (autores, profesores, directores de tesis, pares, etc.) y con artefactos (libros, artículos científicos, reglamentaciones y normativas nacionales e institucionales, etc.) que acarrean la historia de la comunidad de práctica. Así, aprender a escribir una tesis implica adentrarse en los valores y comportamientos de la comunidad académica (Casanave y Li, 2008).

Una de las herramientas que parecen ser fundamentales para poder ajustar los escritos a las expectativas de las comunidades disciplinares parece ser la retroalimentación: no solo ayuda a mejorar los textos (Hattie y Timperley, 2007) sino también atañe y realza el aspecto social de la escritura ya que ocurre en contextos particulares, entre personas que negocian relaciones e identidades particulares (Hyland y Hyland, 2006).
Al respecto, las prácticas de retroalimentación y las relaciones de los tesistas con sus directores o supervisores han sido documentadas (Badenhorst y Guerin, 2016; Basturkmen, East, y Bitchener, 2012; Cadman y Cargill, 2007; Casanave, 2008; Dysthe, 2002; Hyatt, 2005; Kumar y Stracke, 2011; Martín Torres, 2012). De hecho, la importancia de la retroalimentación de los escritos es tal que han surgido propuestas pedagógicas para el nivel de posgrado que proponen expandir el ámbito de injerencia de estas prácticas más allá de la díada supervisor/estudiante (Aitchison y Lee, 2006). De esta manera, se propone abrir oportunidades de participación para los estudiantes mediante dispositivos que permitan interactuar con pares tales como la supervisión grupal, los grupos de estudio o los círculos de lectura/escritura (Aitchison, et al., 2010; Aitchison y Lee, 2006; Kamler y Thomson, 2006). En estos espacios, entonces, los estudiantes ejercerían las prácticas de revisión y discusión de textos borradores $y$, por ende, tendrían oportunidad de mejorar no solo los textos, sino sus hábitos como escritores.

De hecho, algunas investigaciones han mostrado que el trabajo con borradores sucesivos y, sobre todo, las interacciones con pares en el acto de revisión del escrito son importantes ya que refuerzan tanto el acto de escribir para comunicar como el sentimiento de autoría de los estudiantes (Carlino, 2008c; Colombo, 2013; Graff, 2009), incrementan el tiempo invertido en la revisión y las transformaciones del texto (Allal, Lopez, Lehraus, y Forget, 2005), y, finalmente, pueden llevar no solo al mejoramiento del texto, sino también de las prácticas letradas (Alvarez Angulo, 2011; Álvarez Angulo, 2012; Colombo, 2016 ). Sin embargo, estos trabajos son incipientes y el foco de la mayoría de las investigaciones parece seguir estando en el ámbito académico y en aquellas personas con las cuales los doctorandos tienen una relación desigual de poder tal como profesores y directores de tesis. Por ende, no se han tenido en cuenta otras posibles fuentes de retroalimentación o feedback para los escritos intermedios en el proceso de redacción de las tesis.

Así pues, partiendo del anteriormente mencionado enfoque sociocultural del aprendizaje y para lograr una comprensión en profundidad del proceso de elaboración de tesis, nos es necesario identificar los elementos contenidos en la red de relaciones que acompaña al proceso y, simultáneamente, caracterizar las interacciones entre dichos elementos. Es por ello que en este trabajo proponemos expandir la mirada desde los textos y los contextos académicos hacia las interacciones con personas de diferentes ámbitos que influencian el proceso de redacción de tesis. 
Específicamente, exploramos el rol de los lectores prueba: aquellas personas que interactúan con borradores o escritos intermedios relacionados con el trabajo de tesis.

En las siguientes secciones presentamos, en primer lugar, la metodología empleada en esta investigación. Luego, exponemos los resultados del análisis de las entrevistas. Finalmente, en las conclusiones contrastamos nuestros hallazgos con los de anteriores investigaciones y proponemos algunas acciones institucionales dirigidas a acompañar y facilitar el proceso de redacción de tesis por parte de estudiantes de posgrado.

\section{Metodología}

Para abordar el objetivo de nuestro estudio exploratorio, utilizamos entrevistas en profundidad a 20 doctorandos y recientes doctores en el área de Educación y Lingüística, quienes realizaban o habían finalizado recientemente sus estudios en universidades públicas y privadas del área metropolitana de Buenos Aires, Argentina. Estos datos se complementaron con los hallados en documentos y espacios virtuales relativos a la estructura curricular y las pautas institucionales de elaboración de tesis de cada programa, lo cual permitió una puesta en contexto de los mismos. La mayoría de los participantes cursaban doctorados en el área de educación (13 de 20), contaban o contaron con becas parciales o totales para sus estudios (15 de 20), al momento de las entrevistas tenían una edad promedio de 38 años y eran mujeres (13 de 20). La mayoría de los participantes del área de Educación habían cursado maestrías antes de encarar sus estudios doctorales, no sucediendo esto con los de Lingüística.

Las entrevistas fueron transcriptas y para su análisis se siguió una estrategia categorizadora y contextualizadora (Maxwell y Miller, 2008) y se combinaron procesos inductivos y deductivos. Categorías emanadas del marco teórico y la revisión de la literatura (por ej., interacciones con pares y expertos que permiten participar gradualmente en prácticas letradas específicas) guiaron un primer análisis. Estas categorías se reformularon progresivamente con base en el material empírico. Luego de una primera codificación abierta realizada mediante lecturas recurrentes, se identificaron actores y acciones que influían el proceso de tesis en forma negativa o positiva. Estos códigos se refinaron y se obtuvieron categorías más generales y abstractas basadas en los tipos de relaciones y su influencia en el proceso de tesis de los entrevistados. Asimismo, las relaciones entre códigos y categorías fueron analizadas para identificar temas y patrones (Miles y Huberman, 1994).

La triangulación de fuentes de datos y métodos de recolección permitió sumar profundidad y complejidad al proceso de análisis (Denzin, 1989; Tashakkori y Teddlie, 1998). Este último también se enriqueció con la inclusión de esta investigación en el GICEOLEM (Grupo para la Inclusión y Calidad Educativas a través de Ocuparnos de la Lectura y la Escritura en todas las Materias) ya que permitió discutir códigos y categorías y establecer acuerdos inter-jueces, lo cual ayudó a enriquecer y potenciar el análisis de los datos. Finalmente, los integrantes del equipo de investigación colaboraron con la lectura crítica de sucesivas reelaboraciones del presente artículo.

Gracias a esto se diferenciaron entre las relaciones sociales que influencian el proceso de redacción de las tesis, aquellas que pertenecían al ámbito académico, laboral, y familiar de los tesistas. A su vez, al analizar las relaciones sociales mencionadas por los entrevistados, encontramos que la manera en que estas afectan el proceso de tesis muestra una configuración compleja y dinámica.

Al tener en cuenta si han influido de manera negativa o positiva el proceso de tesis, encontramos que la valencia $^{1}$ de los vínculos no es total y estática, sino dinámica y circunstancial. Así, un vínculo con valencia negativa que obstaculiza el proceso de tesis en un momento, puede luego facilitarlo, pasando así a adquirir una valencia positiva; y viceversa. Por ejemplo, al enfermarse el padre de una de las entrevistadas, ella tuvo que mudarse y hacerse cargo de su cuidado lo cual restringió el tiempo que tenía disponible para escribir su tesis. Pero luego, una vez mejorado, su padre fue uno de sus lectores principales de su borrador final y, por lo tanto, colaboró con la corrección del texto. Los resultados que presentamos en esta ocasión surgen de un análisis pormenorizado de las relaciones de todos los ámbitos (laboral, académico, personal) que influenciaban en forma positiva el proceso de tesis de los entrevistados ofreciendo un tipo específico de ayuda académica (Colombo, 2014): actuar como lector prueba.

\section{Resultados}

El análisis de las 20 entrevistas muestra que los doctorandos, además de compartir sus textos intermedios con personas pertenecientes a su ámbito académico y laboral, también pidieron a personas de su esfera personal que actuaran como lectores prueba. El siguiente gráfico presenta los principales hallazgos:

${ }^{1}$ Definimos valencia como el rol positivo o negativo atribuido en determinado momento a las interacciones con determinada persona. 
Tabla 1: Proporción de lectores prueba mencionados por los entrevistados según tipos de relaciones.

\section{Relaciones sociales del ámbiło Tipo de relación}

\section{La persona posee experticia}

\section{Académico y laboral (17/20)} Simetica: companeros de programa de

Simétrica: compañeros de programa de doctorado, de equipos de investigación, de grupos de estudio y/o compañeros de trabajo en
instituciones académicas $(16 / 20)$.

Asimétrica: co-directores, ex directores de tesis, profesores de sus programas doctorales, directores de grupos de investigación ajenos al propio, evaluadores de eventos académicos $y / 0$ mentores informales $(17 / 20)$.

\section{Si $(17 / 20)$ No $(0 / 20)$}

\section{Personal $(12 / 20)$}

Familiares o de pareja: padres, madres, hijas, esposas, novios, ex maridos, hermanos $(12 / 20)$.

Amigos que no están en el ámbito académico (3/20).

Nota. Entre paréntesis se da la proporción de entrevistados que mencionaron recurrir a esas relaciones sociales para que actúen como lectores prueba.

En cuanto a las relaciones sociales del ámbito académico, algunas personas con quienes comparten sus borradores son pares, mientras con otras mantienen relaciones asimétricas.

La mayoría de los entrevistados (16 de 20) mencionaron compartir con frecuencia borradores de sus tesis con pares del ámbito académico. Entre ellos, se mencionaron compañeros de programa de doctorado, de equipos de investigación, de grupos de estudio y/o compañeros de trabajo en instituciones académicas. Por ejemplo, Valeria $^{2}$ declaró que quienes con más frecuencia leían sus textos intermedios eran sus "compañeros"3 , entre los que incluyó personas de su equipo de investigación y de "un grupo de estudios literarios para-institucional" en el que participaba.

Por su lado, Lucía mencionó que sus "compañeros de doctorado" funcionaban como lectores-prueba y detalló: "Bianca me lee un montón. Y en su momento, Omar también (....) entre los tres éramos un buen grupo de apoyo". En forma similar, a Daria le leía sus borradores Ofelia, "una compañera que ya tenía beca y que trabajaba con temas afines" así como también sus compañeras de cursos de doctorado. En definitiva, casi la totalidad de los entrevistados expresaron compartir sus textos intermedios con pares o compañeros quienes ayudaron con la escritura de la tesis al interactuar con textos intermedios y ofrecer comentarios y correcciones que ayuden a mejorar la versión final.
Además de compartir sus borradores con pares, la mayoría de los participantes (17/20) mencionaron recurrir a personas del ámbito académico con quienes no tenían una relación de iguales tales como co-directores, ex directores de tesis, profesores de sus programas doctorales, directores de grupos de investigación ajenos al propio, evaluadores de eventos académicos y/o mentores informales. Por ejemplo, Ana mencionó que mientras escribía su tesis tuvo que lidiar con campos de conocimientos ajenos a su formación por lo que consultó directores e integrantes de otros equipos de investigación ya que "necesitaba como unas orientaciones" y que "alguien me fuera leyendo".

Por su parte, Ariadna mencionó a una profesora de la cátedra en la cual ella era adscripta, quien "oficialmente no figura en los papeles" por lo que no era directora ni co-directora de su tesis, pero sí cumplía una función de mentora. En sus palabras: "hoy por hoy es la persona más presente en lo que es mi trabajo de investigación (...) que está más pendiente de lo que hago, la que más me lee, es ella". Algo similar relató Jimena quien dijo que Belén, una de las profesoras que ella consideraba su mentora, no era su co-directora "no por nada en especial, básicamente para no hacer el trámite" pero era quien más devoluciones le hacía cuando ella le enviaba borradores tal como muestra la siguiente cita:

yo le escribo 10 hojas y ella me devuelve 20 de comentarios. Hay más comentarios que hojas

\footnotetext{
1 Los nombres de los entrevistados y las personas que mencionan han sido cambiados por cuestiones de confidencialidad.

2 Las expresiones entrecomilladas y en itálicas son fragmentos de las transcripciones de las entrevistas. "(...)" indica que parte del discurso se ha elidido. Se señala mediante corchetes "[ ]" la inclusión de aclaraciones para que el lector comprenda el discurso.
} 
que yo mando, pero hace como un seguimiento, que, bueno, te súper acompaña en lo que escribís (...) no es que me lo corrige [el texto], ni siquiera es que lo corrige, sino que te pone preguntas. "¿y esto te parece así, no sería mejor?, ¿no utilizás acá tal cosa?, ¿y esto cómo lo pensás con lo otro?".

Este patrón es consistente con el hecho de que la mayoría de los entrevistados que mencionaron a sus directores no los calificaron como lectores prueba de borradores tempranos, sino como a quienes entregaban versiones finales, es decir, textos que consideraban "acabados" tal como expresa Leonel:

La directora fue Nicasia pero tuve dos grandes lectoras. Tamara. Tamara se recibió de doctora aquí [se refiere a la institución en la cual cursa su doctorado] en el 2007, (...) o sea, ella no podía dirigirme. Pero Tamara (...) leía cada una de las cosas que yo escribía para la tesis. Y fue una lectora fundamental (...) mi otra gran lectora, que es Juana Duran, (...) yo la conocí en un seminario que dio (...) me quedé enganchadísimo [entusiasmadísimo] y ella fue la que me empezó a tirar algunas puntas de cómo yo podía encarar la tesis (...) a Nicasia [su directora] lo que hice fue le di la tesis como más aprontada.

Además de recurrir a personas de su ámbito académico, los datos muestran que los doctorandos solicitan a personas de su ámbito personal que oficien de lectores-prueba de borradores de sus tesis. En efecto, 12 de los 20 entrevistados mencionaron compartir con parejas, familiares y/o amigos borradores de sus tesis. Si bien estos últimos lectores-prueba no pertenecen a los campos disciplinares sobre los que versan las investigaciones, influencian el proceso de escritura de las tesis de diferentes formas.

Por un lado, más de la mitad de los entrevistados que dijeron consultar parejas, familiares y amigos (8 de 12) afirmaron que estas personas les aportaban una mirada "externa" que propiciaba revisiones que les permitían mejorar la escritura de sus tesis. Por ejemplo, Ariel, expresó que la mirada de su mujer como lectora-prueba era valiosa ya que "no está muy metida adentro" por lo cual podía aportarle perspectivas nuevas fuera de sus supuestos disciplinares. En forma paralela, Flavia declaró creer que las devoluciones de su novio sobre sus borradores, a pesar de que no entendiera mucho sobre el tema de su tesis, le eran útiles:

"es alguien [su novio] con quien puedo, que puede ver cosas que yo no, desde una mirada digamos no desde la XX [menciona el nombre del campo disciplinar de su tesis], sino como desde la organización general del texto, desde la comunicación hacia el otro, si doy o no por sentadas cosas, si hay cosas que se entienden, si hay lagunas. Porque yo esto del marco teórico ya lo tengo como reinteriorizado, entonces a veces puede ser que no me dé cuenta de que haya algo importante de explicitar y yo no lo esté explicitando"

Por otra parte, 5 de los 12 entrevistados que dijeron consultar parejas, familiares y amigos declararon contar con lectores-prueba que no pertenecían a su disciplina o ámbito académico, pero poseían cierto nivel de experticia o conocimiento práctico sobre alguna de las cuestiones abordadas en la tesis. Por ejemplo, el ex-marido de Amalia, quien era filósofo, la ayudó con su tesis ya que le facilitó una "lectura más conceptual" de los conceptos lingüísticos que ella utilizaba y Daria pidió a su padre, profesor de historia, que leyera la parte de su tesis donde brindaba un contexto histórico "para ver la bibliografía que estaba manejando".

En definitiva, los datos muestran que los tesistas no solo piden a personas de su ámbito académico que actúen como lectores prueba. Además de compartir sus borradores tempranos con profesores, codirectores, tutores y compañeros de diferente índole, los entrevistados declararon que los comentarios y devoluciones hechos por personas de su ámbito familiar también les resultaban útiles, manejaran o no el tema de su tesis. Por una parte, aquellos que no estaban familiarizados con el campo del conocimiento o el tema abordado por la tesis aportaban correcciones en cuanto a cuestiones de redacción tales como ortografía o cuestiones mecánicas de estilo, así como comentaban sobre la claridad del escrito. Esto se vuelve especialmente relevante en el caso de la tesis, la cual presenta una "naturaleza pseudo-comunicativa" (Shaw, 1991, p. 194) y constituye un "género borroso" (Medway, 2002) que puede consustanciarse de muchas formas. Es decir, este tipo de escrito demanda explicar contenidos a una audiencia experta (por ej., autores reconocidos en la especialidad) y a la vez informar a una audiencia no especialista (por ej., miembros del comité evaluador de la tesis que pertenecen a la comunidad disciplinar, pero no se especializan en el tema). Los lectores-prueba ajenos al ámbito académico facilitarían a los tesistas posibles interpretaciones del texto realizadas por una audiencia que no es especialista en el contenido ni está familiarizada con el género.

Por otra parte, los lectores intermedios del ámbito académico y también aquellos ajenos al ámbito académico pero que contaban con conocimientos disciplinares o algún tipo de conocimiento específico, contribuían al comentar cuestiones del escrito desde 
su calidad de expertos en determinado campo disciplinar, fuere este igual o diferente al de la tesis. Así, la retroalimentación ofrecida por colegas, familiares o amigos ayudaba a los tesistas a revisar, por ejemplo, la solidez de los conceptos, autores y contenidos presentados en sus borradores. Es decir, estas personas oficiaban de "agentes mediadores" (Dysthe, 2002; Lillis y Curry, 2006) ya que "traducían" a los tesistas las expectativas discursivas del género y el campo disciplinar, para que ellos pudieran satisfacerlas en sus escritos.

\section{Conclusión}

Nuestros datos muestran que, en efecto, los lectores prueba son un recurso que los doctorandos utilizan con frecuencia y consideran valioso para el proceso de redacción de las tesis. Estos resultados son consistentes, por un lado, con aquellas investigaciones centradas en las prácticas de retroalimentación de escritos en el ámbito académico y, por el otro, con estudios de caso que analizan el proceso de escritura de tesis.

En primer lugar, las investigaciones centradas en los procesos de supervisión y comentario de las tesis tanto por superiores como por pares (Badenhorst y Guerin, 2016; Boud y Lee, 2005; Carlino, 2008b, 2015; Colombo, 2015; Meschitti, 2018, entre otros) plantean, de la misma manera en la que lo hacen nuestros entrevistados, que compartir textos intermedios con otros ayuda a los doctorandos a desarrollar las capacidades necesarias para poder participar de nuevas comunidades discursivas. Es decir, los doctorandos aprenden a pensar, actuar, escribir y hablar como científicos de determinada comunidad de práctica principalmente a través de prácticas de participación, modelado y ejercicio de prácticas que incluyen interacciones significativas con miembros de la comunidad centradas alrededor de sus producciones textuales. Esto pone de relieve la importancia que las redes de retroalimentación de escritos juegan para aquellos doctorandos que se encuentran atravesando el proceso de redacción de tesis.

En segundo lugar, nuestros hallazgos se encuentran en línea con varios estudios de casos que han mostrado cómo los contextos culturales, laborales y familiares de los que participan los estudiantes también influyen y son influenciados por el desarrollo de sus prácticas letradas (Ferenz, 2005; Hawisher, Selfe, Moraski, y Pearson, 2004; Johanson, 2001; Trice, 2004). Así, nuestro estudio aporta evidencia sobre cómo las relaciones sociales originadas en ámbitos no académicos también juegan un rol en el proceso de desarrollo de la identidad y las prácticas escriturales a nivel de posgrado. Ya fuere por sus aportes en cuanto a su conocimiento conceptual o práctico de determinadas temáticas o a la claridad del escrito, los lectores de textos intermedios del ámbito no académico parecen brindar a los tesistas una ayuda que, al parecer, no resulta de fácil acceso: lectores-prueba dispuestos a compartir impresiones e interpretaciones del texto que permitan mejorarlo (Carlino, 2008a; Colombo y Carlino, 2015).

Estos aportes no suplen, sino que complementan aquellos ofrecidos por los integrantes de la comunidad académica de referencia para los tesistas, con quienes mantienen relaciones de igualdad o no.

Ya fuere con el foco en el ámbito académico o en el ámbito personal de los tesistas, creemos que este conjunto de investigaciones que conceptualizan la escritura de géneros disciplinares como un fenómeno de participación social aportan información sobre los procesos reales de producción textual de las tesis y abren el camino a repensar iniciativas pedagógicas enfocadas en aumentar el acceso a lectores prueba en los diferentes estadios de desarrollo del trabajo de tesis. Al respecto, las instituciones podrían brindar mayor respaldo y estructura a las prácticas de supervisión, así como facilitar la incorporación de los tesistas en equipos de investigación. También podrían llevar a cabo iniciativas que fomenten el trabajo con textos intermedios en instancias tempranas de los programas doctorales. Por ejemplo, se podría incluir la revisión entre pares en los cursos o seminarios que los estudiantes toman desde sus primeros años. Esto no solo les permitiría experimentar la escritura como un proceso gradual, sino también familiarizarse con el aspecto colegial de dar y recibir comentarios.

En la misma línea, se podría impulsar a consultar a personas ajenas a la disciplina y fomentar la formación de grupos de escritura, los cuales han probado ser efectivos para el avance de las tesis doctorales (Aitchison, 2009; Aitchison y Lee, 2006; Colombo, 2013, 2015; Colombo y Carlino, 2015; Ferguson, 2009; Guerin, et al., 2012; Kumar y Aitchison, 2017; Márquez Guzmán y GómezZermeño, 2018; entre otros). Creemos que el desarrollo de iniciativas de este tipo facilitaría el proceso de tesis y contribuiría no solo al aprendizaje de prácticas letradas situadas por parte de los doctorandos, sino también al aumento de las tasas de finalización de los programas de posgrado. 


\section{Referencias}

Ahern, K., y Manathunga, C. (2004). Clutch-starting stalled research students. Innovative Higher Education, 28(4), 237-254.

Aitchison, C. (2009). Writing groups for doctoral education. Studies in Higher Education, 34(8), 905916.

Aitchison, C., Kamler, B., y Lee, A. (2010). Publishing pedagogies for the doctorate and beyond. New York: Routledge.

Aitchison, C., y Lee, A. (2006). Research writing: Problems and pedagogies. Teaching in Higher Education, 11(3), 265-278.

Alvarez Angulo, T. (2011). Revising and rewriting in collaborative writing in higher education and beyond. Journal of Academic Writing, 1(1), 100-109.

Álvarez Angulo, T. (2012). Revisar y reescribir textos académicos en la formación del profesorado. Revista Complutense de Educación, 22(2), 269-294.

Allal, L., Lopez, L., Lehraus, K., y Forget, A. (2005). Whole-class and peer interaction in an activity of writing and revision. In T. Kostouli (Ed.), Writing in context(s): Textual practices and learning processes in sociocultural settings (pp. 69-91). New York: Springer.

Arnoux, E. (Ed.). (2009). Escritura y producción de conocimiento en las carreras de posgrado. Buenos Aires: Santiago Arcos Editor.

Badenhorst, C., y Guerin, C. (Eds.). (2016). Research literacies and writing pedagogies for masters and doctoral writers. Amsterdam: Brill/Emerald Publishing.

Basturkmen, H., East, M., y Bitchener, J. (2012). Supervisors' on-script feedback comments on drafts of dissertations: socialising students into the academic discourse community. Teaching in Higher Education, 1-14.

Boud, D., y Lee, A. (2005). 'Peer learning' as pedagogic discourse for research education. Studies in Higher Education, 30(5), 501-516.
Brunner, J. J., y Ferrada Hurtado, R. (Eds.). (2011). Educación superior en Iberoamérica. Informe 2011. Santiago de Chile: Centro Interuniversitario de Desarrollo (CINDA) - Universia.

Cadman, K., y Cargill, M. (2007). Providing quality advice on candidates' writing. In C. Denholm y T. Evans (Eds.), Supervising doctorates downunder: Keys to effective supervision in Australia and New Zealand (pp. 182-191). Victoria: Acer Press.

Canagarajah, A. S. (2002). A geopolitics of academic writing. Pittsburgh, PA.: University of Pittsburgh Press.

Carlino, P. (2005). ¿Por qué no se completan las tesis en los posgrados? Obstáculos percibidos por maestrandos en curso y magistri exitosos. Educere, 9(30), 415-420. Disponible en http:// www.scielo.org.ve/scielo.php?script=sci_arttextypid $=$ S1316-49102005000300020

Carlino, P. (2008a, septiembre). Desafíos para hacer una tesis de posgrado y dispositivos institucionales que favorecerían su completamiento. Ponencia presentada en el Segundo Encuentro Nacional y Primero Internacional sobre Lectura y Escritura en Educación Superior, Bogotá, Colombia.

Carlino, P. (2008b). Exploración de géneros, diario de tesis y revisión entre pares: Análisis de un ciclo de investigación-acción en talleres de tesis de posgrado. En E. Arnoux (Ed.), Escritura y producción de conocimientos en carrera de posgrado ( $\mathrm{pp}$. 220-239). Buenos Aires: Santiago Arcos Editores. Disponible en https://sites.google.com/site/ jornadasgiceolem/posgrado.

Carlino, P. (2008c). Revisión entre pares en la formación de posgrado. Lectura y Vida, 29(2), 20-31. Disponible en https://sites.google.com/site/jornadasgiceolem/ posgrado.

Carlino, P. (2012). Helping doctoral students of Education to face writing and emotional challenges in identity transition. En M. Castelló y C. Donahue (Eds.), University writing: Selves and texts in academic societies (Vol. 24, pp. 217-234). Bingley: Emerald Group Publishing Limited.

Carlino, P. (2015). Revisión entre pares: Una práctica social que los posgrados deberían enseñar. Espaço Pedagogico, 22(1), 9-29. 


\section{0 • artículos Científicos}

Casanave, C. P. (2008). Learning participatory practices in graduate school: Some perspective-taking by a mainstream instructor. En C. P. Casanave y X. Li (Eds.), Learning the literacy practices of graduate school: Insiders' reflections on academic enculturation (pp. 14-31). Ann Arbor: The University of Michigan Press

Casanave, C. P., y Li, X. (Eds.). (2008). Learning the literacy practices of graduate school: Insiders' reflections on academic enculturation. Ann Arbor: The University of Michigan Press

Colombo, L. (2012). Escritura de posgrado y aprendizaje situado. En Facultad de Psicología de la Universidad de Buenos Aires (Ed.), Memorias del IV Congreso Internacional de Investigación y Práctica Profesional en Psicología - XIX Jornadas de Investigación - VIII Encuentro de Investigadores en Psicología del MERCOSUR [en CD] (Vol. 1, pp. 82-85). Buenos Aires, Argentina: Ediciones de la Facultad de Psicología - Universidad de Buenos Aires. Disponible en https://sites.google.com/site/ jornadasgiceolem/posgrado.

Colombo, L. (2013). Una experiencia pedagógica con grupos de escritura en el posgrado. Aula Universitaria, 15, 61-68. Disponible en http:// bibliotecavirtual.unl.edu.ar/publicaciones/index.php/ AulaUniversitaria/article/view/4368/6643.

Colombo, L. (2014). Los vínculos personales en la producción de tesis doctorales. Revista Electrónica de Investigación Educativa (REDIE), 16(2), 8196. Disponible en http://redie.uabc.mx/vol16no82/ contenido-colombo.html.

Colombo, L. (2015). La escritura en el posgrado: Grupos de escritura en el contexto anglosajón y argentino. En C. Muse (Ed.), Cátedra UNESCO. Lectura y escritura: continuidades, rupturas y reconstrucciones (Vol. 4/Lectura y escritura como prácticas sociales, pp. 249-257). Córdoba: Universidad Nacional de Córdoba.

Colombo, L., y Carlino, P. (2015). Grupos para el desarrollo de la escritura científico-académica: Una revisión de trabajos anglosajones. Lenguaje, 43(1), 13-34. Disponible en http://revistalenguaje.univalle. edu.co/index.php/Lenguaje/article/view/3276.

Denzin, N. (1989). The research act (3ra ed.). Englewood Cliffs, NJ: Prentice Hall.
Dysthe, O. (2002). Professors as mediators of academic text cultures: An interview study with advisors and Master's degree students in three disciplines in a Norwegian university. Written Communication, 19(4), 493-544.

Elgar, F. (2003). PhD completion in Canadian universities. Final Report. Halifax, Nova Scotia: Graduate Students Association of Canada.

Ferenz, O. (2005). EFL writers' social networks: Impact on advanced academic literacy development. Journal of English for Academic Purposes, 4, 339-351.

Ferguson, T. (2009). The 'write' skills and more: A thesis writing group for doctoral students. Journal of Geography in Higher Education, 33(2), 285-297.

Graff, N. (2009). Approaching Authentic Peer Review. The English Journal, 98(5), 81-87.

Guerin, C., Xafis, V., Doda, D. V., Gillam, M. H., Larg, A. J., Luckner, H., et al. (2012). Diversity in collaborative research communities: A multicultural, multidisciplinary thesis writing group in public health. Studies in Continuing Education, 35(1), 65-81.

Hattie, J., y Timperley, H. (2007). The power of feedback. Review of Educational Research, 77(1), 81-112.

Hawisher, G. E., Selfe, C. L., Moraski, B., y Pearson, M. (2004). Becoming literate in the information age: Cultural ecologies and the literacies of technology. College Composition and Communication, 55(4), 642-692.

Hyatt, D. F. (2005). 'Yes, a very good point!': A critical genre analysis of a corpus of feedback commentaries on Master of Education assignments. Teaching in Higher Education, 10(3), 339-353.

Hyland, K., y Hyland, F. (Eds.). (2006). Feedback in second language writing: Contexts and issues. New York: Cambridge University Press.

Johanson, R. E. (2001). The role of interactions in academic writing: A collective case study of five Taiwanese doctoral students in a graduate school of education. Tesis doctoral, The University of Texas at Austin, United States.

Kamler, B., y Thomson, P. (2006). Helping doctoral students write: Pedagogies for supervision. London and New York: Routledge. 
Kumar, V., y Aitchison, C. (2017). Peer facilitated writing groups: a programmatic approach to doctoral student writing. Teaching in Higher Education, 1-14.

Kumar, V., y Stracke, E. (2011). Examiners' reports on theses: Feedback or assessment? Journal of English for Academic Purposes, 10(4), 211-222.

Lave, J., y Wenger, E. (1991). Situated learning: Legitimate peripheral participation. New York: Cambridge University Press.

Lee, I. (2013). Publish or perish: The myth and reality of academic publishing. Language teaching, 47(2), 1-12.

Lillis, T., y Curry, M. J. (2006). Professional academic writing by multilingual scholars. Written Communication, 23(1), 3.

Lovitts, B. E., y Nelson, C. (2000). The hidden crisis in graduate education: Attrition from $\mathrm{Ph}$. D. programs. Academe, 86(6), 44-50. Disponible en http://www. aaup.org/AAUP/pubsres/academe/2000/ND/Feat/ lovi.htm?PF=1

Lundell, D. B., y Beach, R. (2003). Dissertation writers' negotiations with competing activity systems. En C. Bazerman y D. R. Russell (Eds.), Writing selves, writing societies: Research from activity perspectives. Perspectives on writing. (pp. 483514). Fort Collins, CO: The WAC Clearinghouse and Mind, Culture, and Activity.

Márquez Guzmán, S., y Gómez-Zermeño, M. G. (2018). Grupo Virtual de escritura académica. Una e-innovación para impulsar la publicación científica. Revista Mexicana de Investigación Educativa, 23(76), 203-227. Disponible en https:// www.comie.org.mx/v201/revista/visualizador.php?ar ticulo=ART76008ycriterio=http://www.comie.org.mx/ documentos/rmie/v76023/n76076/pdf/76008.pdf.

Martín Torres, G. G. (2012). La escritura de tesis de posgrado en el área de investigación educativa: El acompañamiento, una pieza clave. CPU-E Revista de Investigación Educativa, 15, 69-86.

Maxwell, J. A., y Miller, B. (2008). Categorizing and connecting strategies in qualitative data analysis. En S. Hesse-Biber y P. Leavy (Eds.), Handbook of emergent methods (pp. 461-477). New York: The Guilford Press.
Medway, P. (2002). Fuzzy genres and community identities: The case of architecture students' sketchbooks. En R. Coc, L. Lingard y T. Teslenko (Eds.), The rhetoric and ideology of genre (pp. 123154). Cresskill, NJ: Hampton Press.

Meschitti, V. (2018). Can peer learning support doctoral education? Evidence from an ethnography of a research team. Studies in Higher Education, 1-13.

Miles, M., y Huberman, A. M. (1994). Qualitative data analysis: An expanded sourcebook. Thousand Oaks, CA: Sage.

Nygaard, L. P. (2015). Publishing and perishing: An academic literacies framework for investigating research productivity. Studies in Higher Education, 42(3), 519-532.

Prior, P. (1998). Writing/disciplinarity: A sociohistoric account of literate activity in the academy. Mahwah, N.J.: Lawrence Erlbaum Associates.

Shaw, P. (1991). Science research students' composing processes. English for Specific Purposes, 10(3), 189-206.

Tashakkori, A., y Teddlie, C. (1998). Mixed methodology: Combining qualitative and quantitative approaches. Thousand Oaks, CA: Sage.

Torrance, M., y Thomas, G. V. (1992). The writing experiences of social science research students. Studies in Higher Education, 17(2), 155.

Trice, A. G. (2004). Mixing it up: International graduate students' social interactions with American students. Journal of College Student Development, 45(6), 671-687.

Vygotsky, L. S. (1978). Mind in society: The development of higher psychological processes. Cambridge, MA: Harvard University Press.

Wenger, E. (1998). Communities of practice: Learning, meaning, and identity. New York: Cambridge University Press. 